\title{
O Prêmio Nobel de Física de 2017 e as Instituições Brasileiras de Pesquisas Físicas ${ }^{+*}$
}

José Maria Filardo Bassalo ${ }^{1}$

Academia Paraense de Ciências

Belém - PA

M. Cattani ${ }^{1}$

Instituto de Física - USP

São Paulo - SP

\section{Resumo}

Neste artigo, trataremos do Prêmio Nobel de Física de 2017, concedido aos físicos norte-americanos Rainer ("Rai") Weiss (de origem alemã), que recebeu a metade e, a outra metade foi dividida, por Barry Clark Barish e Kip Stephen Thorne - pelas decisivas contribuições na construção do detector LIGO e a observação de ondas gravitacionais -, segundo o Comitê Nobel (CN). Trataremos, também, dessas ondas e sua detecção, além mostrar como os três nobelistas se relacionaram.

Palavras-chave: Prêmio Nobel de Física de 2017; Weiss; Barish e Thorne; Ondas Gravitacionais; Detecção.

\begin{abstract}
In this article, will be addressed the Nobel Prize in Physics of 2017, awarded to American physicists Rainer ("Rai") Weiss (of German origin), who received half, and the other half was split, by Barry Clark Barish and Kip Stephen Thorne - for the decisive contributions in the construction of the LIGO detector and the observation of gravitational waves - according to the Nobel Committee (CN). We also analyze these
\end{abstract}

\footnotetext{
+ The 2017 Physics Nobel Prize and the Brazilians Institutions of the Physical Researches

* Recebido: dezembro de 2017.

Aceito: abril de 2018.

${ }^{1}$ E-mails: jmfbassalo@gmail.com; mcattani@if.usp.br
} 
waves and their detection, as well we show the relationship between these three nobelists.

Keywords: Nobel Prize of Physics of 2017; Weiss; Barish and Thorne; Gravitational waves; Detection.

\section{Introdução}

O PNF/2017 foi compartilhado pelos físicos norte-americanos Rainer ("Rai”) Weiss (n.1932) (de origem alemã), que recebeu a metade e, a outra metade foi dividida, por Barry Clark Barish (n.1936) e Kip Stephen Thorne (n.1940) - pelas decisivas contribuições na construção do detector LIGO e a observação de ondas gravitacionais -, segundo o Comitê Nobel (CN). Registre-se que as Nobel Lectures, foram por eles apresentadas, no dia 08 de dezembro de 2017, com os títulos: LIGO and Gravitational Waves I (Weiss); LIGO and Gravitational Waves II (Barish); LIGO and Gravitational Waves III (Thorne).

Inicialmente, vejamos um pequeno resumo histórico das ondas gravitacionais (OG) e que será desenvolvido tendo como base os seguintes artigos: Mauro Sérgio Dorsa Cattani, Gravitacional Waves I: Basic Emission Equations [arXiv:1001.2518v1 (gr-qc) (2010)]; Gravitacional Waves II: Basic Emission Systems Equations [arXiv:1003.2105v1 (grqc) (2010)]; e Gravitacional Waves III: Detecting Systems [arXiv:1004.2470v1 (gr-qc) (2010)]; José Maria Filardo Bassalo e Mauro Sérgio Dorsa Cattani, Detecção de Ondas Gravitacionais. http://publica-sbi.if.usp.br/PDFs/pd 1693 (07/04/2016) e Gravitational Waves Observation: Brief Comments. http://publica-sbi.if.usp.br/PDFs/pd 1696 (01/08/2016), Revista Brasileira de Ensino de Física, v. 3, n. 4, Epub (Oct 10, 2016); verbetes da wikipedia.org; e Google Imagens.

Em dezembro de $1915^{2}$, o físico germano-suíço-norte-americano Albert Einstein (1879-1955; PNF, 1921), formulou a hoje famosa Teoria da Relatividade Geral (TRG), traduzida pela também hoje célebre Equação de Einstein (EE):

$$
\mathrm{R}_{\mu \nu}-(1 / 2) \mathrm{g}_{\mu \nu} \mathrm{R}=-\mathrm{K} \mathrm{T}_{\mu \nu},
$$

na qual $\mathrm{R}_{\mu v}$ é o tensor geométrico de Ricci $\left(\mathrm{R}_{\mu \nu}\right), \mathrm{R}=\mathrm{g}^{\mu v} \mathrm{R}_{\mu \nu}, \mathrm{g}_{\mu \nu}=\mathrm{g}^{\mu v}$ é o tensor métrico riemanniano, $\mathrm{K}=8 \pi \mathrm{G} / \mathrm{c}^{4}$ é a constante gravitacional de Einstein, sendo $\mathrm{G}$ a constante de gravitação universal de Newton-Cavendish, c é a velocidade da luz no vácuo, e $\mathrm{T}_{\mu \nu}$ é o tensor energia-matéria.

No começo de 1916³ , o astrônomo alemão Karl Schwarzschild (1873-1916) encontrou uma solução [conhecida como a Métrica de Schwarzschild (MS)] para a EE e definida pela expressão:

\footnotetext{
2 Sitzungsberichte Preussische Akademie der Wissenschaften 2, p. 778; 799; 831; 844, 1915.

${ }^{3}$ Sitzungsberichte Preussische Akademie der Wissenschaften 1, p. 189, 1916.
} 


$$
\mathrm{ds}^{2}=(1-2 \mathrm{mG} / \mathrm{R}) \mathrm{c}^{2} \mathrm{dt}^{2}-[1 /(1-2 \mathrm{mG} / \mathrm{R})] \mathrm{dr}^{2}-\mathrm{r}^{2}\left(\mathrm{~d} \theta^{2}-\operatorname{sen}^{2} \theta \mathrm{d} \varphi^{2}\right),
$$

onde $\mathrm{m}$ é a massa de uma partícula puntiforme colocada em um campo gravitacional isotrópico e estático e $(r, \theta, \varphi)$ representam as coordenadas esféricas. Por essa expressão vê-se, claramente, que quando $\mathrm{R}=2 \mathrm{~m} \mathrm{G}$, há uma singularidade de ds, isto é: ds $\rightarrow \infty$. Esse valor fícou conhecido como o Raio de Schwarzschild $\left(\mathrm{R}_{\mathrm{Sch}}\right)$ o que corresponde a conhecida Singularidade de Schwarzschild (SS).

Em junho de $1916^{4}$, Einstein obteve uma solução aproximada da EE, ao considerar campos gravitacionais fracos e, como resultado dessa consideração, concluiu pela existência de ondas gravitacionais (OG). Ainda nesses trabalhos, Einstein tentou calcular a radiação gravitacional (RG) (de comprimento de onda $\lambda$ ) emitida por um sistema mecânico isolado excitado (sendo $<\mathrm{v}>$ a velocidade média de suas partículas internas), com dimensões lineares $\mathrm{r}$ ( $\mathrm{r}<<$ $\lambda)$, e no regime não relativista $(<v><<c)$. Em 19185, Einstein voltou a calcular a RG, ocasião em que corrigiu um erro que havia cometido em 1916 sobre o mesmo tema, e apresentou sua célebre fórmula da RG decorrente da perda de energia mecânica (E). Em notação atual, essa Fórmula do Quadrupolo de Einstein (FQE) é dada por:

$$
\mathrm{dE} / \mathrm{dt}=\left[\mathrm{G} /\left(5 \mathrm{c}^{2}\right)\right]\left[\Sigma_{\mathrm{i}, \mathrm{j}=1,2,3}\left(\mathrm{~d}^{3} \mathrm{Q}_{\mathrm{ij}} / \mathrm{dt}^{3}\right)\right]^{3},
$$

onde $\mathrm{Q}_{\mathrm{ij}}=\int \rho\left[\mathrm{x}_{\mathrm{i}} \mathrm{x}_{\mathrm{j}}-(1 / 3) \delta_{\mathrm{ij}} \mathrm{r}^{2}\right] \mathrm{d}^{3} \mathrm{x}$ é o momento de quadrupolo, $\rho$ é a densidade da fonte gravitacional, $\delta_{\mathrm{ij}}$ é o tensor de Kronecker, $\mathrm{r}^{2}=\mathrm{x}^{2}+\mathrm{y}^{2}+\mathrm{z}^{2}$ e sendo $\mathrm{d}^{3} \mathrm{x}=\mathrm{dx} d \mathrm{dz}$ (lembrar que: $\left.x_{1}=x, x_{2}=y, x_{3}=z\right)$. Em 19376, Einstein e o físico norte-americano Nathan Rosen (1909-1955) estudaram as ondas gravitacionais cilíndricas como solução da EE.

É oportuno salientar que uma solução de ondas gravitacionais planas da EE foi encontrada, em 19577 , pelo astrofísico austro-inglês Sir Hermann Bondi (1919-2005). Ainda em 19578, os físicos norte-americanos John Archibald Wheeler (1911-2008) e Joseph Weber (1919-2000) analisaram as ondas gravitacionais cilíndricas de Einstein-Rosen. Por outro lado, as soluções de ondas planas exatas das OG foram encontradas, em 19599, por Bondi, e os físicos ingleses Felix Arnold Edward Pirani (1928-2015) e Ivor Robinson (1923-2016). Em trabalhos independentes realizados, ainda em 1959, os físicos norte-americanos Richard Lewis Arnowitt (1928-2014) e Stanley Deser (n.1931)10 e Paul Adrien Maurice Dirac (1902-1984; PNF, 1933) ${ }^{11}$ desenvolveram um formalismo hamiltoniano não-covariante da gravitação para

\footnotetext{
${ }^{4}$ Sitzungsberichte Preussische Akademie der Wissenchaften 1, p. 423; 688, 1916.

${ }^{5}$ Sitzungsberichte Preussische Akademie der Wissenchaften 1, p. 154, 1918.

${ }^{6}$ Journal of the Franklin Institute, v. 223, p. 43, 1937.

7 Nature, v. 179, p. 1072, 1957.

${ }^{8}$ Reviews of Modern Physics, v. 29, p. 509, 1957.

${ }^{9}$ Proceedings of the Royal Society of London A251, p. 519, 1959.

${ }^{10}$ Physical Review, v. 113, p. 745, 1959.

11 Physical Review, v. 114, p. 924, 1959.
} 
calcular amplitudes de transição da RG. Como Dirac, em 1958 ${ }^{12}$, havia iniciado esse formalismo [completado em 195913], ele passou a ser conhecido como Universo de Dirac.

A ideia de construir um equipamento para medir as OG foi apresentada por Weber, em 196014, constituído de grandes cilindros de alumínio (Al). Em 1961 e 196215, o astrônomo germano-norte-americano Rainer ("Ray") Kurt Sachs (n. 1932) estudou as OG no espaçotempo plano assintótico. Também, em 1962 ${ }^{16}$, Bondi, M. G. J. van der Burg e A. W. K. Metzner estudaram as OG de sistemas isolados com simetria axial. Em 1963, os físicos norteamericanos Julian Seymour Schwinger (1918-1994; PNF, 1965) ${ }^{17}$ e Richard Philips Feynman (1918-1988; PNF, 1965)18 usaram a Teoria de Campos para quantizar o campo gravitacional (gráviton). Por sua vez, as OG foram estudadas pelo astrofísico norueguês Sjur Refsdal (1935-2009), em 196419. Ainda em 1964²0, o físico norte-americano Steven Weinberg (n.1933; PNF. 1979) estudou a probabilidade de emissão de OG usando a Mecânica Quântica. Em 196521, o físico russo Vladimir Borisovich Braginsky (1931-2016) discutiu como detectar as OG. Em 1966²2, em 196723, em 196824, em $1969^{25}$ e, em 197026, Weber voltou a descrever a construção de grandes cilindros de $\mathrm{A} \ell$ para detectar as $\mathrm{OG}$.

Nesta oportunidade, é interessante destacar que a detecção das OG se intensificou com a descoberta dos pulsares. Vejamos como ocorreu essa descoberta. Em agosto de 1967, a astrônoma irlandesa Susan Jocelyn Bell Burnell (n.1943), então estudante do radioastrônomo inglês Antony Hewish (n.1924; PNF, 1974), encontrou objetos celestes, na nebulosa de Caranguejo, que emitiam vibrações regulares de ondas de rádio. Ao comunicar essa descoberta a Hewish, os dois então pensaram que haviam realizado contato com uma civilização extraterrestre, razão pela qual deram o nome de Little Green Men (LGM1) ("Pequenos Homens Ver-

\footnotetext{
12 Proceedings of the Royal Society of London A246, p. 333, 1958.

13 Physical Review, v. 114, p. 924, 1959.

14 Physical Review, v. 117, p. 307, 1960.

15 Proceedings of the Royal Society of London A264, p. 309; A270, p. 103, 1962.

16 Proceedings of the Royal Society of London A269, p. 21, 1962.

17 Physical Review, v. 130, p. 1253, 1963.

18 Acta Physica Polonica, v. 24, p. 697, 1963.

${ }^{19}$ Monthly Notices of the Royal Society of London, v. 128, p. 295, 1964.

${ }^{20}$ Physics Letters, v. 9, p. 357; Physical Review B135, p. 1049; B140, p. 516, 1964.

21 Uspekhi Fizika Nauk, v. 86, p. 433, 1965.

22 Physics Review Letters, v. 17, p. 1228, 1966.

23 Physics Review Letters, v. 18, p. 498, 1967.

24 Physics Review Letters, v 20, p. 1307, 1968.

25 Physics Review Letters, v. 2.2, p. 1320, 1969.

26 Physics Review Letters, v. 25, p. 180, 1970.
} 
des"). No entanto, a análise mais detalhada dessa observação levou Hewish e sua equipe (Burnell, J. H. D. Pilkington, Paul F. Scott e R. A. Collins) a anunciar, em $1968^{27}$ a descoberta de uma estrela da ordem da massa solar e de raio da ordem de $10 \mathrm{~km}$, e que gira em torno de si com um período de $\approx 1,337 \mathrm{~s}$. Note-se que essa estrela recebeu o nome de CP 1919, onde CP significa Cambridge Pulsar e 1919 indica sua posição nos céus. Denote-se que, em 196828, Weber discutiu a possibilidade de o pulsar NP 0532, na nebulosa de Caranguejo, ser um emissor de OG. Em $1969^{29}$ e em 19700, Weber anunciou que havia encontrado evidências experimentais da $\mathrm{RG}$, pois observou a coincidência de pulsos dessa radiação em cilindros de $\mathrm{A} \ell$ colocados a uma distância de 1.000 km, entre o College Park, em Maryland, e o Argonne National Laboratory, em Illinois. A partir dessa proposta de Weber, foi desenvolvida a técnica de detectores ressonantes, que usa a oscilação de sólidos muito rígidos (geralmente na forma cilíndrica e esférica) para detectar as OG [Cattani (2010), op. cit.].

Em dezembro de 1973, o astrofísico norte-americano Russell Alan Hulse (n.1950; PNF, 1993) foi trabalhar no Arecibo Radio Telescope, em Porto Rico, operado pela Cornell University, na frequência de $430 \mathrm{MHz}$, no qual havia registros de cerca de 100 pulsares até então conhecidos. Seu objetivo era o de preparar sua Tese de Doutoramento sob a orientação do astrofísico norte-americano Joseph Hooton Taylor Junior (n.1941; PNF, 1993) que ensinava na University of Massachusetts, especialista em pulsares, pois, em 197231, juntamente com Richard N. Manchester e G. R. Huguenin desenvolvera um algoritmo de dispersãocompensação para estudar 22 pulsares. Entre dezembro de 1973 e janeiro de 1975, Hulse descobriu 40 novos pulsares. Contudo, um deles, observado no dia 02 de julho de 1974, na constelação de Águia, não se enquadrava na crença geral de que esses objetos celestes eram estrelas de nêutrons solitárias e girantes. Esse pulsar, denominado de PSR $1913+16$, onde PSR significa pulsar e o número é a sua posição no céu, apresentava um período de $0,05903 \mathrm{~s}$. Contudo, no dia 25 de agosto de 1974, Hulse tentou obter um período mais acurado para esse pulsar. Depois de realizar um ajuste do efeito Doppler (1842)-Fizeau (1848) devido ao movimento da Terra, Hulse encontrou uma diferença de $27 \mu \mathrm{s}\left(1 \mu \mathrm{s}=10^{-6} \mathrm{~s}\right)$ em suas medidas. Em princípio, pensou tratar-se de uma falha em seu programa de computador, o ZBTREE. Reescreveu-o e voltou a observar o PSR 1913 + 16, entre 01 e 02 de setembro de 1974 . Novamente encontrou uma variação do período com o tempo, variação essa que permaneceu nas observações subsequentes. Então, no dia 18 de setembro de 1974, escreveu uma carta para Taylor, que se encontrava em Amherst, dizendo-lhe que o PSR $1913+16$ era um pulsarbinário, com período de $\sim 8$ horas. Desse modo, Hulse e Taylor, em Arecibo, começaram a

\footnotetext{
${ }^{27}$ Nature, v. 217, p. 709, 1968.

${ }^{28}$ Physics Review Letters, v. 21, p. 395, 1968.

${ }^{29}$ Physical Review Letter, v. 22, p. 1320, 1969.

${ }^{30}$ Physics Review Letters, v. 24, p. 276; 25, p. 180, 1970.

${ }^{31}$ Nature-Physical Science, v. 240, p. 74, 1972.
} 
realizar uma análise desse objeto celeste usando as leis de Kepler, encontrando os seguintes dados: velocidade orbital $\sim 300 \mathrm{~km} / \mathrm{s}$, tamanho da órbita da ordem do raio do Sol $\left(\mathrm{R}_{\mathrm{S}}\right)(=6,96$ $\left.\times 10^{8} \mathrm{~m}\right)$, massas do sistema binário da ordem da massa solar $\left(\mathrm{m}_{\mathrm{S}}=1,99 \times 10^{30} \mathrm{~kg}\right)$, e período de 7 h 45 min. Essa descoberta foi anunciada em $1974^{32}$ e em $1975^{33}$. Registre-se que foi nesses artigos que Hulse e Taylor afirmaram que esse objeto celeste poderia servir para testar as OG, pois qualquer sistema que radiasse esse tipo de onda perderia energia. Assim, em virtude dessa perda de energia, as estrelas do pulsar-binário se aproximariam uma da outra e, em consequência, haveria uma diferença em seu período orbital. Considerando essa possibilidade, Taylor e colaboradores passaram a verificar se havia essa diferença no período do PSR 1913 + 16. O primeiro resultado dessa variação foi anunciado, em 197934, por Taylor, Lee A. Fowler e Peter M. McCulloch. Mais tarde, em 198235, Taylor e Joel M. Weisberg apresentaram o seguinte resultado: $(2,40 \pm 0,09) \times 10^{-12} \mathrm{~s} / \mathrm{s}$, sendo o valor teórico einsteniano de: $(2,403 \pm 0,02) \times 10^{-12} \mathrm{~s} / \mathrm{s}$. Esse resultado levou Taylor a fazer o seguinte comentário: - Portanto, 66 anos depois de Einstein prever as ondas gravitacionais, um experimento foi realizado e que apresenta clara evidência de sua existência. Essa concordância entre experiência e teoria foi confirmada por Taylor em $1992^{36}$. Observe-se que antes, em 1991, Taylor ${ }^{37}$ mostrou que os pulsares milissegundos (períodos entre 1 e $10 \mathrm{~ms}$ ) descobertos e estudados por ele são os relógios naturais mais estáveis do Universo, e que Taylor e o físico francês Thibault Damour (n.1951) ${ }^{38}$ investigaram a mudança do período orbital do PSR $1913+16$. É interessante registrar que, em $1992^{39}$, Alexander Wolszczan (n.1942) e Dale A. Frail (n.1961) anunciaram a descoberta de um sistema de dois planetas, três vezes mais massivos do que a Terra, girando em torno do pulsar milissegundo PSR 1257 + 12. Note-se que Wolszczan, em 1994 (Science 264, p. 538), anunciou a existência de um terceiro planeta girando em torno desse PSR $1257+$ 12, com um período de 25,34 dias.

Após 30 anos de pesquisas verificou-se que os detectores sólidos são muito pouco eficientes e que se alguma OG fosse detectada seria um evento fortuito. Assim, a partir de 1990 esses detectores deixaram de ser usados e começaram a ser substituídos por dispositivos que usam a interferência das ondas (em geral ondas de luz, rádio ou som), como, por exemplo: 1) Interferômetro de Michelson-Morley (IM-M), inventado pelo físico norte-americano

\footnotetext{
32 Astrophysical Journal Letters, v. 191, p. L59, 1974.

33 Astrophysical Journal, v. 195, p. L51; Astrophysical Journal Letters, v. 201, p. L55, 1975.

34 Nature, v. 277, p. 437, 1979.

35 Astrophysical Journal, v. 253, p. 908, 1982.

${ }^{36}$ Philosophical Transactions of the Royal Society of London 341, p. 117, 1992.

${ }^{37}$ Proceedings of the Institute of Electrical and Electronic Engineers (IEEE), v. 79, p. 1054, 1991.

38 Astrophysical Journal, v. 366, p. 501, 1991.

${ }^{39}$ Nature, v. 355, p. 145, 1992.
} 
físico Albert Abraham Michelson (1852-1931; PNF, 1907), em $1881^{40}$ e melhorado pelo e químico norte-americano Edward William Morley (1838-1923), em 188741; e 2) Interferômetro (Cavidade) de Fabry-Pérot [(I)CF-P], idealizado pelos físicos franceses Charles Fabry (1867-1945) e Jean Baptiste Gaspard Alfred Pérot (1863-1925), em 189842]. Basicamente, esses aparelhos (para o caso da luz) são constituídos de um divisor de feixe (espelho semiprateado) e dois espelhos $\left(\mathrm{E}_{1} / \mathrm{E}_{2}\right)$ colocados na extremidade de braços em forma de L. Depois de refletidos $\left(E_{1} / E_{2}\right)$ eles passam de novo pelo divisor e se encontram em um detector que formará um padrão de interferência, padrão esse que permitirá calcular a diferença de tempo da luz entre os dois braços. Com a invenção do primeiro laser óptico, em $1960^{43}$, pelo físico norteamericano Theodore Harold Maiman (1927-2007), a precisão desses instrumentos foi significantemente aumentada.

Uma primeira ideia sobre a construção de um Laser Interferometer GravitationalWave Observatory (LIGO) ("Observatório de Ondas Gravitacionais por Interferômetro Laser" $)^{44}$ e cujo objetivo fundamental era o de observar as OG de origem cósmica, deve-se a Weiss, em 1968, quando estava no Massachusetts Institute of Technology (MIT) (onde entrou, em 1964, e permanece lá até hoje). Quase vinte (20) anos depois, em 01 de junho de $1987^{45}$, os cientistas Andrew D. Jeffries, Peter R. Saulson, Robert E. Spero e Michael E. Zucker publicaram o artigo intitulado Gravitational Wave Observatories, no qual discutiram a construção de observatórios (tipo LIGO) para detectar as OG. Por fim, o LIGO foi proposto pelos cientistas norte-americanos Rochus E. ("Robbie") Vogt, Ronald ("Ron") William Prest Drever (1931-2017), Thorne e Frederick J. Raab, que trabalhavam no California Institute of Technology (CALTECH) e mais Weiss (MIT), em 1989, à National Science Foundation (NSF), dos Estados Unidos da América, proposta essa que foi detalhada por eles [e mais outros cientistas: Alex Abramovici, William E. Althouse, Yekta Gursel, Seiji Kawamura, Lisa Sievers, Robert E. Spero, Stanley Ernest Whitcomb (n.1951) e Zucker, do CALTECH, e David H. Shoemaker, do MIT], em um artigo publicado, em 17 de abril de $1992^{46}$.

Por sua vez, na Europa, a ideia de detectar as OG partiu de pesquisadores de seis países (França, Itália, Holanda, Polônia, Hungria e Espanha), sob a liderança da França e da Itália, por intermédio do Centre National de la Recherche Scientifique (CNRS) ("Centro Nacional de Pesquisa Científica”), em 1993, e do Istituto Nazionale di Física Nucleare (INFN) (“Instituto Nacional de Física Nuclear"), em 1994, ao criarem o Detector Virgo, nome deriva-

\footnotetext{
40 American Journal of Sciences, v. 22, p. 120, 1881.

41 American Journal of Sciences, v. 34, p. 333, 1887; Philosophical Magazine, v. 24, p. 449, 1887.

42 Comptes Rendus Hebdomadaires des Séances de l’Académie des Sciences, v. 126, p. 34, 1898.

43 Nature, v. 187, p. 493, 1960; British Communications Electronics, v. 1, p. 674, 1960.

$44<$ en.wikipedia.org/LIGO>.

45 Scientific American, v. 256, p. 50, 1987.

46 Science, v. 256, p. 325, 1992.
} 
do do fato de que tais cientistas queriam observar o Virgo Cluster ("Aglomerado de Virgem"), com cerca de 1.500 galáxias da Virgo Constellation ("Constelação de Virgem"), distante cerca de $50 \times 10^{6}$ anos-luz da Terra.

Agora, vejamos a construção desses detectores e suas atualizações (avanços). Segundo registramos acima, o LIGO foi proposto a NSF, em 1989, por uma colaboração entre alguns cientistas do CALTECH e do MIT, com seus detalhes publicados em 1992 (Science, op. cit.). Uma vez que essa construção envolvia milhões de dólares, sua viabilidade econômica deveu-se, fortemente, ao nobelista Barish [que havia se bacharelado, em 1957, na University of California/Berkeley e se doutorado também nessa Universidade, em 1965, com a Tese intitulada Searches for New Physics Using High Mass Dimuons at CDF II Experiment, sob a orientação oficial do físico norte-americano August Carl Helmholz (1915-2003)]. Em 1963, juntou-se ao CALTECH, para fazer parte do famoso grupo de Física Experimental de Alta Energia, que havia sido criado pelo físico-norte-americano Ernest Orlando Lawrence (19011958; PNF, 1939), no começo da década de 1930. Como Barish tornou-se um cientista de alto prestígio científico, embora não tenha participado do grupo inicial do LIGO, em 1994 ele tornou-se seu principal investigador e conseguiu, neste mesmo ano, a aprovação do projeto LIGO pela NSF e, para dar seguimento à construção do LIGO, Barish criou, em 1997, a LIGO Scientific Collaboration (LSC) e indicou Weiss para ser seu primeiro spokeperson ("portavoz"). Note-se que, antes, em 1996, o CNRS e o INFN, iniciaram a viabilização do Virgo ${ }^{47}$.

O LIGO foi construído em 1999 e opera com dois observatórios (em forma de L, com $4 \mathrm{~km}$ de comprimento de lado) para detectar as OG: o Hanford Observatory, do Hanford Nuclear Reservation, próximo de Richland, Washington (WA), e o Livingston Observatory, em Livingston, Los Angeles (LA), separados por $3.002 \mathrm{~km}$, que corresponde a $10 \mathrm{~ms}$ (1 ms = $10^{-3} \mathrm{~s}$ ) na chegada das OG, uma vez que esta viaja, teoricamente, com a velocidade da luz no vácuo $(c=300.000 \mathrm{~km} / \mathrm{s}$ ). Observe-se que o LIGO, que é composto dos interferômetros IMM e (I)CF-P, começou a operar em 23 de agosto de 2002, mas somente foi finalizado em 2010 por dificuldades orçamentárias.

Em dezembro de 2000, o CNRS e o INFN criaram o European Gravitational Observatory (EGO), para promover a pesquisa em gravitação, na Europa, sendo um de seus objetivos a construção e a atualização do Virgo localizado em Cascina, próximo de Pisa, na Itália. Esse detector possui um IM-M, tendo $3 \mathrm{~km}$ cada um de seus lados.

Em 2007, os detectores LIGO e Virgo resolveram compartilhar os resultados de seus trabalhos realizados por intermédio da LIGO e Virgo Collaborations, correspondentes às três detecções das OG, resultantes da fusão de buracos-negros (BN) e de uma quarta decorrente da fusão de um par de estrelas de nêutrons, tema principal do PNF/2017. Vejamos essas detecções, cujos detalhes podem ser vistos no en.wikipedia.org.

$47<$ en.wikipedia.org/Virgo>. 


\section{Primeira detecção (GW150914)}

Por ocasião de uma Conferência realizada na NSF, em Washington, D.C., em 11 de fevereiro de 2016, o físico norte-americano David Reitze (n.1961), Diretor Executivo do LIGO anunciou que duas Colaborações Internacionais (LIGO Scientific Collaboration e VIRGO Collaboration) haviam observado (desde 14 de setembro de 2015) Ondas Gravitacionais (OG) provindas da fusão (merger) de um binário de buracos-negros $(\mathrm{BN})$ com cerca de $62 \mathrm{M}_{\odot}\left(\mathrm{M}_{\odot}\right.$ $=$ massa do Sol $\left.\approx 2 \times 10^{30} \mathrm{~kg}\right)\left(\approx 12,4 \times 10^{31} \mathrm{~kg}\right)$ [sobre os BN, ver: Bassalo e Cattani, 2016 (op. cit.)] e a uma distância de $\approx 1,2 \times 10^{9}$ anos-luz $\left(\approx 1,2 \times 10^{25} \mathrm{~m}\right)$ da Terra, evento esse conhecido como GW150914. Note-se que essas OG ("ondas no espaço-tempo") produziram expansões e contrações nos lados dos dois LIGOS da LSC (cujos braços estariam em posição favorável para a medida, inclusive com uma frequência de $250 \mathrm{~Hz}$ pouco antes do merger), registradas em cada um de seus detectores (um em Hanford e o outro em Livingston) e com uma diferença de tempo de $7 \times 10^{-3}$ s. \{Para detalhes sobre como ocorrem os registros nos detectores, ver: Cattani [arXiv:1004.2470v1 (gr-qc)] (op. cit.)\}. É interessante destacar que este resultado corresponde ao confidence limit ("limite de confiança"): CL > 5,1 $\sigma$ (99,9999\%, significando ser menor do que um evento em cada 203.000 anos). Assim, em 12 de fevereiro de 2016, esse trabalho foi publicado na Physical Review Letters 116, article number (a.n.) 061102, com o título: Observation of Gravitational Waves from a Binary Black Hole Merger, com 1.011 autores [incluindo os três nobelistas: Weiss (LIGO/MIT), Barish (LIGO/CALTECH) e Thorne (CALTECH RELATIVITY THEORY GROUP)], pertencentes a 133 Instituições de Pesquisas Internacionais (p. ex.: Centros, Institutos e Universidades), com destaque para duas brasileiras: 1) Instituto Nacional de Pesquisas Espaciais, em São José dos Campos, em São Paulo (INPE/SP) e 2) Instituto de Física Teórica/Universidade Estadual Paulista (IFT/UNESP) em colaboração com o Salam International Centre for Theoretical Physics/South American Institute for Fundamental Research (SICTP/SAIFR), de São Paulo.

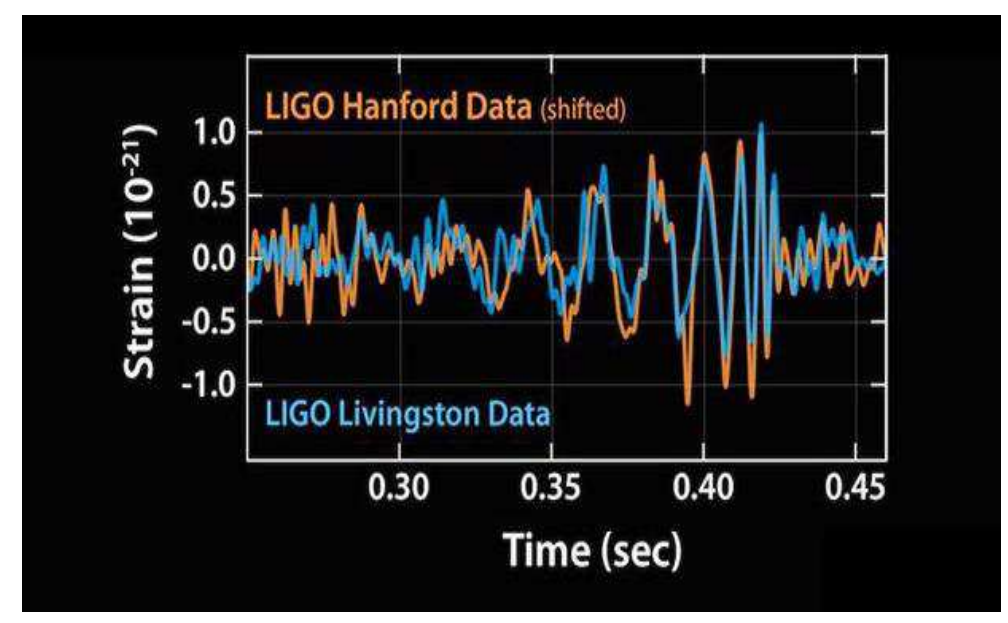

Fig. 1 
Saliente-se que do INPE/SP, participaram cinco físicos brasileiros: Odylio Denys Aguiar (n. 1953); César Augusto Costa; Márcio Constâncio Junior); Elvis Camilo Ferreira e Allan Douglas dos Santos Silva, sob as liderança de Aguiar e Costa. Por sua vez, no IFT/UNESP-SICTP/SAIFR, foram os físicos, o iraniano Saeed Mirshekari e o italiano Riccardo Sturani (n. 1974).

Neste instante, é oportuno destacar que existe outra interpretação para o evento (GW150914). Vejamos qual. Com uma visão instantânea de 70\% do céu e 0,4 s depois do mesmo, o Gamma-ray Burst Monitor (GBM) [pertencente ao Fermi Gamma-ray Space Telescope (Fermi-GRST), levado pelo foguete DELTA II7920-H e lançado pela NASA, em 11 de junho de 2008], detectou a presença de uma fonte fraca de energia eletromagnética acima de $50 \mathrm{keV}$ e com a duração de $1 \mathrm{~s}$. No artigo publicado pelo GBM [arXiv:1602.03920v3 (astroph.HE)], em seu Abstract, seus 29 principais pesquisadores dizem que essa observação parece não estar conectada com nenhuma outra atividade astrofísica (solar, terrestre ou magnetosférica). Porém, por ser consistente com a direção do GW150914, tal observação sugere que tenha ocorrido uma erupção de raios gama $(\gamma)$ e, portanto, incompatível com a fusão de buracos negros. Em vista disso, esses pesquisadores passaram a calcular a luminosidade (L) correspondente a emissão de raios-X duros, entre $1 \mathrm{keV}$ e $10 \mathrm{MeV}$, e encontraram que: $\mathrm{L}=\left[1,8^{+1,5}\right.$. $\left.{ }_{1,0} \times 10^{49}(\mathrm{erg} / \mathrm{s})\right]$. Esse valor, ainda segundo os autores, tem uma confiabilidade de $75 \% \mathrm{e}$, mais ainda, assumindo que os dois eventos (LIGO/GBM) sejam correspondente ao mesmo GW150914, somente uma colaboração futura desses dois observatórios possa reduzir a $90 \%$ o intervalo de confiança numa locação do céu de 601 para $199 \mathrm{deg}^{2}$. Observe-se que $\operatorname{deg}^{2}[\equiv$ $\left({ }^{0}\right)^{2}$ ] ("graus quadrados") é uma medida de ângulo sólido, dado pela expressão: $(\pi / 180)^{2} \approx$ $3,0462 \times 10^{-4}$ esferoradianos. Registre-se que a associação desse evento com a emissão celeste de $\gamma$ foi descartada, pois ele foi associado com uma atividade terrestre. (Sturani, e-mail 14/01/2018).

\section{Segunda detecção (GW151226)}

Uma segunda observação de OG foi registrada pelos dois detectores- avançados do LIGO (Hanford e Livingston) em 26 de dezembro de 2015. Esse evento, denominado de GW151226, teve seu sinal detectado identicamente ao do evento GW150914 e foi gerado pela coalescência de um sistema binário formado por dois buracos negros $(\mathrm{BN})$ com massas $\approx 14,2$ $\mathrm{M}_{\odot} \mathrm{e} \approx 7,5 \mathrm{M}_{\odot}$ (sendo sua massa total de $\approx 20,8 \mathrm{M}_{\odot}$ ), tendo um dos componentes um spin > 0,2 . A fonte de OG estaria localizada a uma distância de $\approx 1,3 \times 10^{9}$ anos-luz $\left(\approx 1,3 \times 10^{25} \mathrm{~m}\right)$ da Terra. A onda detectada durou cerca de $1 \mathrm{~s}$ e a frequência das ondas variou de 35 a $450 \mathrm{~Hz}$, e apresentou um $\mathrm{CL}>5 \sigma$.

Em 15 de junho de 2016, essa detecção foi publicada na Physical Review Letters 116, a.n. 241103, com o título: Observation of Gravitational Waves from a 22-Solar-Mass Binary Black Hole Coalescence, com 979 autores [incluindo os nobelistas: Weiss (LIGO/MIT) e Barish (LIGO/CALTECH)], pertencentes a 141 Instituições de Pesquisas Interna- 
cionais, ainda com a participação dos mesmos físicos do INPE/SP (Aguiar, Constantino Junior, Costa, Ferreira e Santos Silva) e do IFT/UNESP-SICTP/SAIFR (Mirshekari e Sturani).

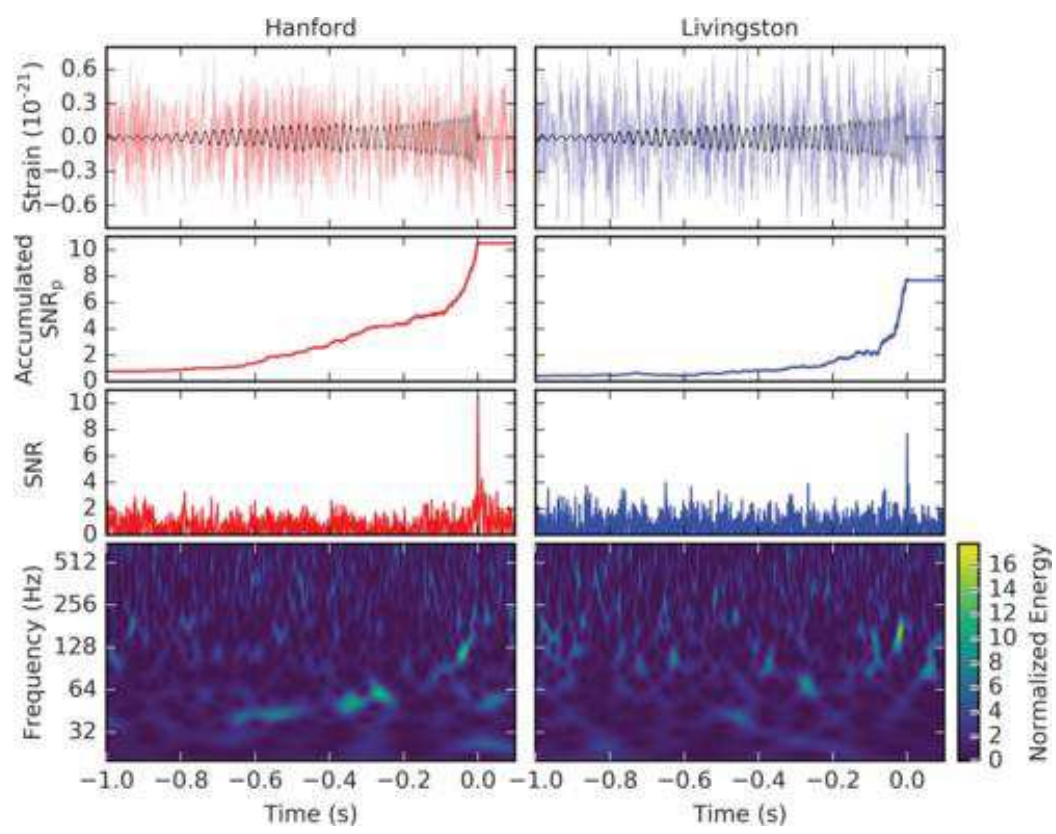

Fig. 2

\section{Terceira detecção (GW170814)}

Uma terceira detecção das OG foi realizada no dia 14 de agosto de 2017 pelos dois detectores-avançados do aLIGO (Hanford e Livingston/USA) e pelo detector-avançado do adVIRGO (Cascina/Itália), em um evento denominado de GW170814. As equipes dos três detectores [que observaram uma região de $60 \mathrm{deg}^{2}$ do Universo usando uma Técnica de Triangulação (TT) e que lhe permitiram, pela primeira vez, testar a natureza de polarização das OG] acreditam que as OG detectadas foram emitidas durante os momentos finais da fusão (coalescência) de dois $\mathrm{BN}$, um com uma massa $\approx 31 \mathrm{M}_{\odot}$, o outro com $\approx 25 \mathrm{M}_{\odot}$. Esse evento, localizado a $\approx 1,8 \times 10^{9}$ anos-luz $\left(\approx 1,8 \times 10^{25} \mathrm{~m}\right)$ da Terra e que resultou em um buraco negro rotativo $(\mathrm{BNR}) \mathrm{com} \approx 53 \mathrm{M}_{\odot}$, o que significa que $\approx 3 \mathrm{M}_{\odot}$ foram convertidas em energia de OG durante a fusão, apresentou uma taxa de alarme-falso de $\lesssim 1$ em 27.000 anos.

O PNF/2017 foi anunciado pelo CN em 03 de outubro de 2017 e, logo depois, em 06 de outubro, essa terceira detecção foi publicada na Physical Review Letters 119, a.n. 141101, com o título: A Three-Detector Observation of Gravitational Waves from a Binary Black Hole Coalescence, com 1.108 autores [incluindo os nobelistas: Weiss (LIGO/MIT) e Barish (LIGO/CALTECH)], pertencentes a 160 Instituições de Pesquisas Internacionais, além dos físicos do INPE/SP (Aguiar, Costa, Constantino Junior, Ferreira, Santos Silva e Marcos André Okada) e de Sturani, agora pertencendo à outra Instituição Brasileira: o International Institute of Physics da Universidade Federal do Rio Grande do Norte (IIP/UFRGN). 

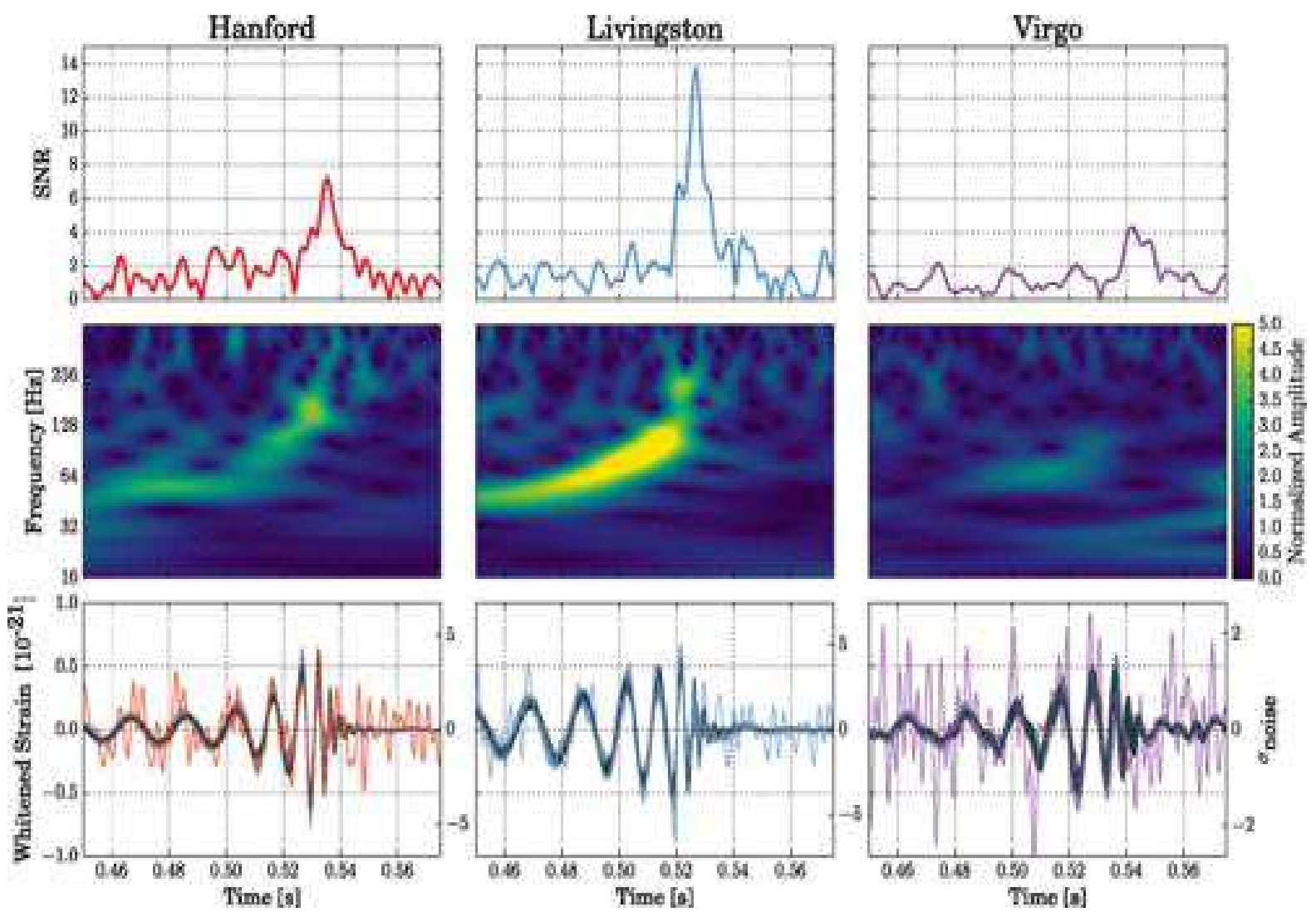

Fig. 3

\section{Quarta detecção (GW170817)}

Uma quarta detecção, registrada no dia 17 de agosto de 2017, além de confirmar os resultados das três detecções anteriores (sobre as $\mathrm{OG}$ devido à fusão de $\mathrm{BN}$ ), anunciou uma descoberta inédita: a fusão de um par de estrelas de nêutrons (EN) produziu, além de OG, ondas electromagnéticas (OEM) em várias frequências: rádio, radiação luminosa visível, infravermelho, ultravioleta, raios-X duros e raios $\gamma$. Vejamos como isso aconteceu.

Assim, naquele dia (17), o GBM anunciou ao mundo que havia identificado um sinal decorrente de uma erupção de raios gama $(\gamma)$ e seguida de uma explosão violenta, chamada então de kilonova, que ocorreu em uma região no céu do hemisfério Sul, na galáxia NCG 4993, na direção da constelação de Hydra, sinal esse oriundo da fusão de um binário de objetos celestes altamente massivos. Note-se que a Collaboration aLIGO/adVirgo detectou uma OG longa e com a duração de $\approx 100 \mathrm{~s}$, com seu pico gravitacional 1,7 s antes da detecção do GBM. Imediatamente vários observatórios no mundo (em terra e no espaço) começaram a investigar esse evento. Por exemplo, aquela Colaboração, ainda no dia 17 (agosto), usou seus dados em conjunto para estudar esse evento. Realizado esse estudo, elas comunicaram, no dia 16 de outubro, que haviam publicado na Physical Review Letters 119, a.n. 161101, o artigo 
intitulado: Observation of Gravitational Waves from a Binary Neutron Star Inspiral. Em seu Abstract, seus autores afirmaram que o sinal, que recebeu a denominação de GW170817, apresentava uma razão de sinal-ruído de 32,4 e com um alarme-falso < 1 em 80.000 anos. Eles encontraram que o componente massivo do binário celeste estava no intervalo de $(0,86$ 2,26) $\mathrm{M}_{\odot}$, de acordo com as massas conhecidas de estrelas de nêutrons (EN). Além do mais, também inferiram que as massas dos componentes do binário variavam de $(1,17-1,60) \mathrm{M}_{\odot}$,

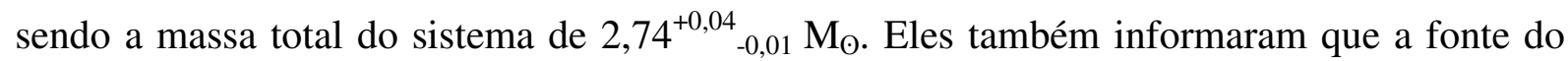
evento estava localizada em uma região do céu de $28 \mathrm{deg}^{2}$ ( $90 \%$ de probabilidade) e sua luminosidade se encontrava a uma distância de $40^{+8}{ }_{-14} \mathrm{Mpc}\left(\approx 0,13 \times 10^{9}\right.$ anos-luz $)$. Note-se que esse artigo foi assinado por 1.096 autores, incluindo os nobelistas: Weiss, Barish e Thorne, pertencentes a 162 Instituições de Pesquisas Internacionais, além da participação dos físicos do INPE/SP (Aguiar, Costa, Constantino Junior, Ferreira, Santos Silva e Okada) e de Sturani, do IIP/UFRGN.

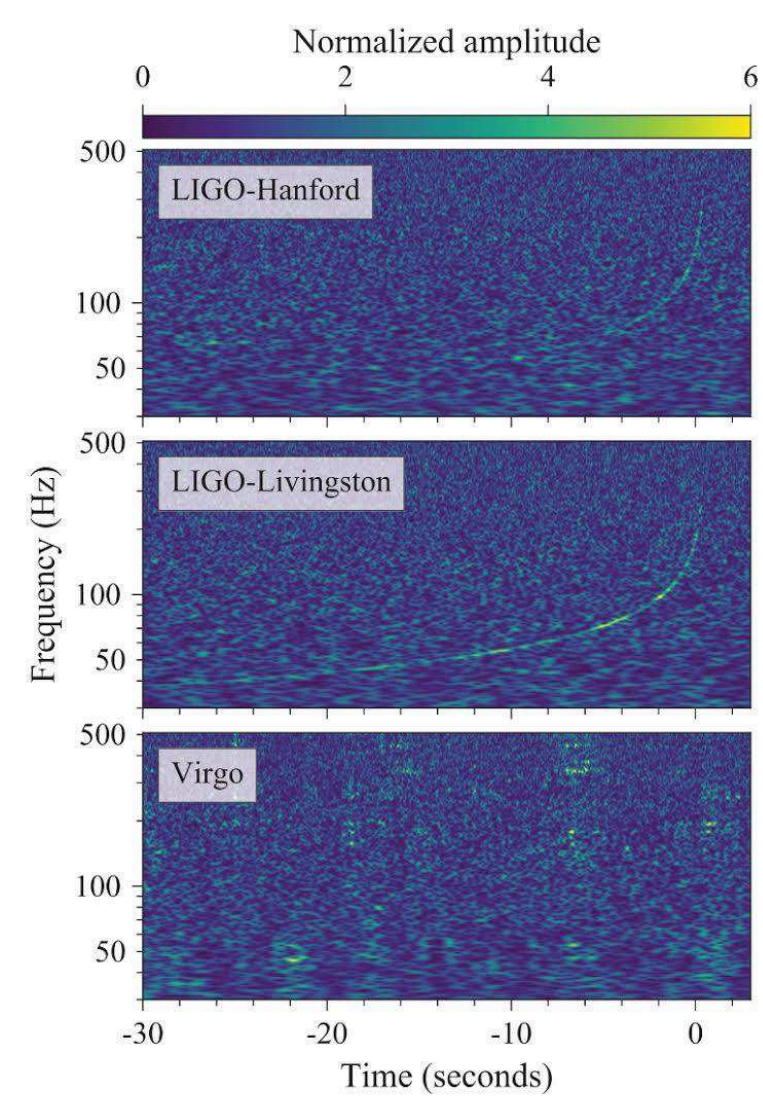

Fig. 4

Como a investigação desse notável evento fora estudado por mais de 3.000 cientistas espalhados por 950 Instituições de Pesquisas Físicas no mundo inteiro (muitas delas no Brasil), os trabalhos resultantes dessa investigação foram apresentados em jornais científicos, como, além do PRL, também o The Astrophysical Journal Letters (AJP), a Nature, a Nature Astronomy e o Science. 
Por exemplo, o AJP 848 (2), de 20 de outubro de 2017, publicou 22 artigos. O de nome Multi-Messenger Observations of a Binary Neutron Star Merger, foi realizado por 58 instituições de pesquisa mundiais. Dentre elas, além do aLIGO/adVirgo, tratado neste artigo, ainda fizeram parte dele três outros Observatórios, os quais também tiveram a participação de outros astrofísicos brasileiros, além dos pertencentes ao aLIGO/adVIRGO, conforme veremos a seguir ${ }^{48}$.

Os três outros Observatórios (além do aLIGO/adVirgo) envolvidos no espetacular evento sobre a detecção de OG pela colisão de EN, são: 1) Observatório Pierre Auger (OPA); 2) Dark Energy Source (DES); e 3) Transient Robotic Observatory of the South (TOROS) ${ }^{49}$.

O OPA foi idealizado em 1992 pelos físicos, o norte-americano James Watson Cronin (1931-2016; PNF, 1980) e o escocês Alan Andrew Watson (n.1938). Ele faz parte de um projeto internacional que reúne cerca de 250 pesquisadores, entre físicos e engenheiros de 55 instituições mundiais (de vários países do mundo, dentre os quais temos a Argentina e o Brasil) e com o objetivo de observar raios cósmicos acima de $10^{19} \mathrm{eV}$ ( $1 \mathrm{eV}$ é a energia eletrostática de um elétron sob a diferença de potencial de 1 Volt). Em novembro de 1995, representantes dessas instituições se reuniram na sede da UNESCO, em Paris, e escolheram a Argentina como local para construir um observatório no hemisfério Sul. Assim, no começo de 1999, foi iniciada sua construção em uma região do oeste argentino (Mendoza), aos pés da Cordilheira dos Andes no deserto de El Nihuil ou Pampa Amarilla, constituindo-se no OPA-SUL.

A participação do Brasil no OPA-SUL envolve as seguintes Instituições Brasileiras de Pesquisas Físicas (IBPF) e correspondentes físicos [AJP 848 (2), L12]: 1) Centro Brasileiro de Pesquisas Físicas (CBPF) - Shellard e Ugo Gregorio Giaccari; 2) Universidade Federal do Rio de Janeiro (UFRJ): 2a) Instituto de Física - Giaccari, Carla Brenda Bonifazi e João Torres de Mello Neto; e 2b) Observatório do Valongo - Mello Neto e C. Ventura; 3) Instituto de Física da Universidade de São Paulo (IF/USP) - Ivone Freire da Mota e Albuquerque, Edivaldo de Moura Santos, Níkolas Kemmerich e W. Rodrigues de Carvalho; 4) Universidade de São Paulo, Instituto de Física de São Carlos (USP/UFSC) - Luiz Vítor de Souza, R. G. Lang e Raul Ribeiro Prado (n.1990); 5) Instituto de Física Gleb Wataghin da Universidade Estadual de Campinas (IFGW/UNICAMP) - Carola Dobrigkeit Chinellato (líder do grupo), José Augusto Chinellato, Anderson C. Fauth, B. Daniel, Mary Lucia Díaz Castro, Márcio Aparecido Muller e L. A. S. Pereira; 6) Universidade de São Paulo, Escola de Engenharia de Lorena (USP/EEL) - Fernando Catalani e Carlos José Todero Peixoto; 7) Universidade Federal do Paraná (UFPR) - R. C. dos Anjos; 8) Universidade Federal de Feira de Santana (UFFS) Germano Pinto Guedes; 9) Universidade Federal do ABC (UFABC) - Marcelo Leigui de Oliveira; 10) Centro Federal de Educação Tecnológica "Celso Suckow da Fonseca" - Bruno La-

\footnotetext{
48 Nesta oportunidade, agradecemos aos físicos Liacir dos Santos Lucena (n.1941) e Sturani, do IIP/UFRGN, assim como Francisco Caruso (n.1959) e Ronald Cintra Shellard (n,1948), do CBPF (e atual Diretor), e João Carlos dos Anjos (n.1944) (também do CBPF e atual Presidente do ON), pelas informações que permitiram o complemento deste artigo.
}

49 Ver detalhes desses observatórios no site: <en.wikipedia.org>. 
zarotto Lago; 11) Universidade Federal de Alfenas, em Minas Gerais (UNIFAL-MG) - Muller; e 12) Universidade Federal Fluminense, Escola de Engenharia Industrial Metalúrgica de Volta Redonda (EEIMEVR) - Rogério Menezes de Almeida e J. de Oliveira. É interessante registrar que o físico brasileiro Carlos Orivio Escobar (n.1948) também participou dessa experiência, porém com o vínculo apenas do FERMILAB.

Vejamos agora o DES e a participação brasileira [AJP 848 (2), L16; L17]. Com o objetivo de mapear o céu do hemisfério sul, a Association of Universities for Research in Astronomy (AURA) (um consórcio sem fins lucrativos) e fundada em 10 de outubro de 1957 e com o apoio da NSF, começou, em 1959, a escolher uma região das montanhas dos Alpes, no Chile, para construír Observatórios Astronômicos (OA) e realizar aquele mapeamento. Em 1962, foi escolhida a Região Coquimbo $(\approx 80 \mathrm{~km}$ a leste de La Serena), que possui montanhas alpinas, dentre elas a Cerro Tololo $(\approx 2.220 \mathrm{~m})$ e a Cerro Pachón $(\approx 2.700 \mathrm{~m})$. No ano seguinte, em 1963, a AURA criou o Cerro Tololo Inter-American Observatory (CTIO) e iniciou a construção de um OA com um espelho de 4.0 m de diâmetro, com suas regulares observações astronômicas [principalmente o grande deslocamento de aglomerados (clusters) de galáxias] iniciadas em 1965. Em 1967, o astrônomo porto-riquenho Victor Manuel Blanco (19182011), foi escolhido seu diretor (até 1980) e, em 1974, concluiu o hoje famoso Telescópio Victor Blanco (CTIO/TVB).

É oportuno destacar que, dentre alguns clusters galáticos, existe o intrigante problema relacionado com sua composição e grande velocidade. Com efeito, em 1934, os astrônomos, o alemão Walter Baade (1893-1960) e o búlgaro-suíço-norte-americano Fritz Zwicky (1898-1974), publicaram artigos nos Proceedings of the National Academy of Sciences 20 (p. 254; 259) e na Physical Review 45 (p. 138), nos quais formularam o conceito de estrela de nêutron (neutron star) (EN) como sendo a estrela cuja massa vale aproximadamente a massa do Sol $\left(\mathrm{M}_{\odot}\right)$, com raio $(\mathrm{R}) \mathrm{de} \approx 10 \mathrm{~km}$ e densidade $(\rho)$ em torno de $\approx 10^{14} \mathrm{~g} / \mathrm{cm}^{3}$. Logo depois, em 193750, ao examinar o aglomerado Coma (que estudava desde 1933), Zwicky fez uma descoberta espetacular: a hoje ainda incompreendida matéria/energia escura (matter/dark energy), que compõe $\approx 95 \%$ (sendo $\approx 70 \%$ de dark energy) do conteúdo do Universo e que é responsável pela expansão do Universo. Esta foi observada, em 199851, por uma equipe (20 cientistas) do High-Z Supernova Search Team (H-ZSST) [criado pelos astrofísicos norteamericanos Brian P. Schmidt (n.1970; PNF, 2011) e Nicholas B. Suntzeff (n.1952), em 1994] e do qual participava (junto com seus criadores) o também astrofísico norte-americano Adam Guy Riess (n.1967; PNF, 2011). Aquela expansão foi confirmada, em 1999, em trabalhos independentes, realizado por outra equipe (42 cientistas) do H-ZSST, ainda com a presença de Riess, Schmidt e Suntzeff ${ }^{52}$, e pelo Supernova Cosmology Project (SCP) (32 cientistas), que

\footnotetext{
${ }^{50}$ Astrophysical Journal, v. 86, p. 217, 1937.

51 Astronomical Journal, v. 116, p. 1009, 1998.

52 Astronomical Journal, v. 117, p. 707, 1999.
} 
pertence ao Lawrence Berkeley National Laboratory (LBNL), liderado pelo astrofísico norteamericano Saul Perlmutter (n.1959; PNF, 2011) ${ }^{53}$. Com a comprovação da existência dessa enorme região escura do Universo, surgiu a necessidade de mapeá-la e assim foi criado o Dark Energy Survey (DES), que é uma colaboração internacional entre vários países (inclusive o Brasil, a partir de 2007).

Como esse mapeamento se destinava a observar minuciosamente milhares de milhões de galáxias, essa colaboração necessitava de detectores de alta sensibilidade. Note-se que esses sensores digitais [Charge-Coupled Device (CCD) - "Dispositivo de CargaEmparelhada"] haviam sido idealizados, em 1970, pelos físicos e engenheiros norteamericanos Willard Sterling Boyle (1924-2011; PNF, 2009) (nascido no Canadá) e George Elwood Smith (n.1930; PNF, 2009) (Bell Systems Technical Journal, Briefs 49, p. 587). Esses dispositivos digitais foram também construídos, ainda em 1970, por Smith, associado com os físicos e engenheiros, o norte-americano Gilbert Frank Amelio (n.1943) e o inglês Michael Francis Tompsett (n.1939) ${ }^{54}$. Por sua vez, em 1981, a Eastman Kodak Company, usou tais dispositivos digitais para inventar a câmara digital (CD).

Essa nova tecnologia (CD) foi importante para utilizá-la em Observatórios Astronômicos (OA), principalmente para investigar a DES. Assim, surgiu a Dark Energy Camera (DECam). A construção da CD (uma câmera de 570 megapixels) foi financiada pele Departamento de Energia dos Estados Unidos da América e contou com a participação de diversas instituições norte-americanas, dentre elas o Berkeley Laboratory, o Argonne National Laboratory, o National Accelerator Laboratory, da Stanford University e o Fermi National Accelerator Laboratory (FERMILAB). A partir de 2004, o FERMILAB começou a desenvolver o projeto do DECam (Telescope Simulator), com sua construção iniciada em 2008 e completada em 2011, para ser adaptada aos OA instalados no hemisfério sul, como o TVB e o Southern Astrophysical Research (SOAR). Este, com 4,1 m de diâmetro, teve sua construção iniciada em 2003, por intermédio de um consórcio incluindo vários países (p. ex.: Brasil, Chile e Estados Unidos) e está localizado na Cerro Pachón. Observe-se que o DECam teve uma participação importante da física brasileira Marcelle Soares-Santos (n.1981) [atualmente professora na Brandeis Unibersity e coordena um grupo, com oito (8) trabalhos publicados no AJP 848 (2), e que procura emissões luminosas associadas à produção de OG (en.wikipedia.org)], pois, ao concluir seu doutorado na Universidade de São Paulo (USP), em 2010 e, ainda neste ano, iniciou seu pós-doutoramento no FERMILAB que estava, como vimos acima, construindo o DECam.

Em vista de que o DECam estava na fase final de sua construção, então, também em 2010, com o apoio do Observatório Nacional (ON), do Laboratório Nacional de Computação Científica (LNCC), e da Rede Nacional de Ensino e Pesquisa (RNP), foi criado o Laboratório Interinstitucional de e-Astronomia (LIneA) com a missão de estimular e coordenar a partici-

\footnotetext{
53 Astrophysical Journal, v. 517, p. 565, 1999.

${ }^{54}$ Bell Systems Technical Journal, v. 49, p. 593, 1970; Applied Physics Letters, v. 17, p. 111, 1970.
} 
pação de pesquisadores brasileiros em grandes observatórios astronômicos (como o TVB e o SOAR). Destaque-se que a DECam foi instalada no TVB entre janeiro de 2011 e 12 de setembro de 2012, dia este que marca a instalação oficial da mesma. Para processar, visualizar e analisar as imagens obtidas pela DECam, entre setembro e outubro de 2012, a LIneA desenvolveu o software [Quick Reduce (QR)] para o CTIO e que foi disponibilizado para uso internacional a partir do primeiro dia do mês de novembro de 2012.

A participação do Brasil no DES, iniciada em 2007, se dá por intermédio do DESBRAZIL, que é um consórcio formado por pesquisadores e técnicos ligados a diferentes IBPF. Contudo, no evento da colisão de EN que estamos tratando, apenas duas delas participaram: 1) ON/LInea - Aurélio Carnero Rosell, Flávia Sobreira (professora-doutora do IFGW/UNICAMP), Júlia Gschwend, Luiz Alberto Nicolaci da Costa (coordenador do DESBRAZIL), Marcos Vinicius Borges Teixeira Lima (professor do IF/USP), Márcio Antonio Geimbra Maia e Ricardo Lourenço Correia Ogando; e 2) Observatório Valongo da Universidade Federal do Rio de Janeiro (OV/UFRJ) - Paulo Afrânio Augusto Lopes (professordoutor/UFRJ) e Ana Carolina Costa Lourenço (mestranda/UFRJ).

Trataremos, agora, da participação das IBPFs no TOROS [AJP 848 (2), L29]. Este projeto astronômico decorreu de uma colaboração entre cientistas da The University of Texas, em Brownsville, da Universidad Nacional de Córdoba, na Argentina, do CALTECH e da Texas A\&M University. Dessa colaboração, resultou a construção de telescópios robóticos em Tolar Grande, em Salta, na Argentina, na Cerro Macón $(\approx 4.650 \mathrm{~m})$, a partir de $2012 \mathrm{e}$, em 2013, ocorreu seu primeiro workshop. Em 2015, a colaboração TOROS concluiu o maior telescópio do planeta, com o espelho refletor de $42 \mathrm{~m}$ de diâmetro, com o qual foi observada a colisão de EN, ocorrida em agosto de 2017. Essa observação contou com a participação de IBPFs e respectivos cientistas, assim registrados: 1) Instituto de Astronomia, Geofísica e Ciências Atmosféricas da Universidade de São Paulo (IAG/USP) - Claudia Lucia Mendes de Oliveira, William Schoenell, Paula R. T. Coelho, Marcus Vinicius Costa-Duarte, Alberto Molino Benito, Laura Maria Sampedro Hernández e Laerte Sodré Junior; 2) Departamento de Física da Universidade Federal de Sergipe (DF/UFS) - T. Ribeiro e Raimundo Lopes de Oliveira Filho; 3) Departamento de Física da Universidade Federal de Santa Catarina (DF/UFSC) - Schoenell e Antônio Kanaan; 4) Departamento de Física Matemática do Instituto de Física da Universidade de São Paulo (DFM/IF-USP) - Luis Raul Abramo; 5) Departamento de Astronomia do Observatório Nacional (DA/ON) - Stravos Akras, Jailson Souza de Alcaniz e Yolanda Jiménez-Tema; e 6) Observatório de Valongo da Universidade Federal do Rio de Janeiro (OV/UFRJ) - Denise Rocha Gonçalves, Thiago Signorini Gonçalves e Laurie Anne Riguccini.

Cremos ser oportuno destacar a participação dos cientistas brasileiros com o Telescópio Brasileiro T80-Sul, localizado no CTIO. Denote-se que este observatório decorreu de um projeto idealizado por Cláudia Mendes (IAG/USP), em 2010, financiado em sua maior parte pela Fundação de Amparo à Pesquisa do Estado de São Paulo (FAPESP), mas também 
pelo ON, e pelas Universidades: USP, UFS e UFSC, sendo o mesmo operado, a partir de 2016, por intermédio do Southern Photometric Local Universe Survey (S-PLUS), constituído por uma colaboração de astrofísicos do Brasil, do Chile e da Espanha. Dentre os brasileiros, além dos já citados, cremos ser necessário incluir, pelo menos mais dois do IAG/USP: seu diretor Pedro Leite da Silva Dias e Rodrigo Nemmen.

Na conclusão deste artigo, vejamos como ocorreu o envolvimento dos três nobelistas $^{55}$. Weiss nasceu em 29 de setembro de 1932, em Berlim, capital da Alemanha. Seu pai era o médico, neurologista e psicanalista alemão Frederick Ernest Weiss (1865-1953), de origem judia e membro ativo do Partido Comunista Alemão; sua mãe era a atriz (cristã) alemã Gertrude Loesner. Quando esta ainda estava grávida de "Rai", seu pai foi preso pelos nazistas [por uma questão menor (testemunha de uma operação malfeita realizada por um médico Nazista), mas certamente por ser judeu e comunista] e depois de ser liberado, por ação política da Família Loesner, ele foi para Praga, capital da Tchecolosváquia. Logo depois do nascimento de "Rai", sua mãe o levou para junto de seu pai, em Praga. Contudo, em setembro de 1938, quando a Ditadura Nazista do político alemão Adolf Hitler (1889-1945) anexou a Tchecolosváquia, exilados judeus que moravam neste país (inclusive a família de "Rai"), correram para os consulados em Praga para obter vistos em seus passaportes e conseguir sair da Tchecolosváquia, pois agora estava totalmente sob o domínio Nazista. Então, o pai de "Rai" foi ao Consulado Norte-Americano para tentar emigrar para lá, em virtude de sua grande relação profissional com a Família Stix, uma proeminente família judia norte-americana, sediada em St. Louis, Missouri, que estava resgatando os profissionais judeus perseguidos pelo Nazismo. Assim, o pai de "Rai" conseguiu o visto para os Estados Unidos e, desde modo, a Família Weiss foi para a Nova York, chegando lá em janeiro de 1939.

Em Nova York, Weiss cresceu no lado oeste superior de Manhattan, frequentando escolas públicas dessa cidade. Quando era adolescente, na segunda metade da década de 1940, ele recebeu uma Bolsa de Estudos da Columbia Grammar and Preparatory School, onde completou o High School, em 1950. Nessa época, ele se interessou por eletrônica, em vista de haver adquirido (em fins de 1946), por preços bem baixos, na Rua Cortland, na parte mais baixa de Manhattan, vários componentes elétricos e eletrônicos (motores, transformadores, tubos de vácuo, capacitores etc.) que sobraram da participação americana na Segunda Guerra Mundial (1939-1945), compras essas que lhe ajudaram bastante em sua "profissão" de consertador de rádios. Nesse início de "pós-guerra", Weiss e amigos (filhos também de imigrantes) assistiam consertos de música clássica no Brooklyn (ao ar livre), bem como na New York Philharmonic e no Carnegie Hall. Nessas audições alguns problemas eram frequentes, como os silvos que apareciam quando sons de uma música clássica apresentavam tons baixos e suaves, assim como dos microfones dos locutores. Sendo um “especialista” em sistemas de áudio, Weiss era convocado para resolvê-los. Contudo, estando no final do ensino médio, ele percebeu que não tinha conhecimentos suficientes de Matemática e de Engenharia Elétrica

$55<$ en.wikipedia.org/RainerWeiss_Autobiography>. 
para resolvê-los. Assim, depois de sondar algumas universidades técnicas norte-americanas, decidiu entrar, em 1950, no Massachusetts Institute of Technology (MIT), escolhendo o Curso de Engenharia Elétrica, cuja estrutura curricular dos dois primeiros anos não o agradou, pois não incluía a parte de eletrônica. Em vista disso, no final do segundo ano ele se transferiu para o Curso de Física. Enquanto aluno do MIT, ele se envolveu emocionalmente com uma aluna de Música da Northwestern University, em Evanston, Illinois, que lhe ensinou a gostar de música folclórica e de piano e, também, com uma aluna do MIT. A distância entre esses dois namoros (Boston e Evanston) ocasionou o fim deles. Além do mais, esse "imbróglio" teve como consequência sua reprovação no MIT, em 1952.

No verão de 1953, com o objetivo de aprender eletrônica, Weiss procurou um emprego de técnico no Research of Laboratory of Electronics (RLE), do MIT, e teve a sorte de encontrar-se com o físico norte-americano Jerrold Reinach Zacharias (1905-1986) que realizava pesquisas com feixes atômicos, buscando construir um relógio atômico usando transições hiperfinas do césio ${ }_{55} \mathrm{Cs}$ ). Depois de conhecer a formação científica de Weiss, os dois começaram a trabalhar nesse relógio tendo como fim medir o redshift gravitacional (mudança da frequência de um átomo radioativo em queda livre) previsto pela Teoria da Relatividade Geral Einsteiniana (TRGE). Eles trabalharam por cerca de dois anos nesse projeto do qual resultou uma versão prática do ainda hoje usado Relógio Atômico a Feixe Efusivo de Césio. Os trabalhos de Weiss com Zacharias renderam-lhe o Bacharelado em Física, em 1955, e o Doutorado em Física, em 1962, com a Tese Intitulada: Stark Effect and Hyperfine Structure of Hydrogen Fluoride, na qual ele calculou a estrutura hiperfina e o momento de dipolo elétrico dos estados de rotação da molécula de fluoreto de hidrogênio (HF). Enquanto trabalhava na Tese, entre 1960 e 1962, Weiss deu aulas na Tufts University.

Obtido o título de Doutor pelo MIT, Weiss iniciou, ainda em 1962, um pósdoutoramento com o físico norte-americano Robert Henry Dicke (1916-1997), na Princeton University (PU), que havia desenvolvido, em 196156, com o físico norte-americano Carl Henry Brans (n.1935), uma Teoria Escalar da Gravitação (TEG), rival da TGRE, segundo a qual a força gravitacional é mediada por um campo escalar. Então, usando a TEG, Dicke e Weiss prepararam um experimento para medir os modos de vibração da Terra, decorrentes da ação de uma provável OG atingindo o nosso planeta. Contudo, esse experimento não conseguiu seu objetivo em virtude da interferência das ondas geradas pelo grande terremoto ocorrido no dia 27 de março de 1964, no Alaska. Então, no outono de 1964, Weiss retornou ao RLE, no MIT, para continuar seus estudos sobre Cosmologia e Gravitação, focados na Radiação Cósmica de Fundo (RCF) (esta decorrente do Big-Bang) e nas OG. Porém, como seu conhecimento matemático da TRGE era insuficiente para esses estudos, ele propôs, em 1967, ministrar um curso dessa teoria para alunos que realizavam a graduação e a pós-graduação no MIT.

\footnotetext{
${ }^{56}$ Physical Review, v, 124, p. 925, 1961.
} 
Por essa época, os relativistas estavam interessados no trabalho que Weber estava desenvolvendo para medir as OG, constituído de grandes cilindros de alumínio (A $)$, decorrente de uma ideia que tivera em 1960. Assim, em 196957, ele anunciou que havia encontrado evidências experimentais de OG, oriunda do pulsar NP 0532, na nebulosa de Caranguejo, pois observou a coincidência de pulsos dessa radiação gravitacional em cilindros de Al (pulsos decorrentes da mudança nos tamanhos desses cilindros pela passagem da OG), que estavam colocados a uma distância de 1.000 km, entre o College Park, em Maryland, e o Argonne National Laboratory, em Illinois.

Em vista dessa informação, logo no começo da década de 1970, Weiss e seus estudantes começaram a trabalhar em uma ideia que ele tivera, em 1968, que era a de medir as OG, por intermédio de um interferômetro do Tipo Michelson-Morley (IM-M) (1887), usando um laser óptico. Assim, em 1972, Weiss solicitou uma verba do RLE (que era destinada pelos militares para o desenvolvimento de pesquisa básica) para construir (como tema de doutorado de seus estudantes) um protótipo desse equipamento, tendo 1,5 $\mathrm{m}$ de lado. Weiss, no entanto, sabia que esse protótipo dificilmente detectaria as OG. Desse modo, ele precisava construir novos protótipos com braços maiores. Porém, em virtude da Guerra do Vietnã (iniciada em 1959), as verbas militares para pesquisa foram cortadas e, então, Weiss apresentou sua ideia para a National Science Foundation (NSF). Esta levou certo tempo para aceitá-la, pois consultou vários especialistas sobre o assunto. Dentre esses especialistas, estavam grupos do Max Planck Institute for Astrophysics e do Max Planck Institute of Quantum Optics, em Garching, na Alemanha, em Garching, na Alemanha, que trabalhavam com a proposta de Weber e que acharam ser a Interferometria Óptica (IO) uma ótima alternativa para detectar as OG. Entraram em contato com Weiss para trabalharem juntos o que aconteceu com a ida de alunos de Weiss para lá. Dessa parceria (com financiamento da NSF), foram construídos mais dois protótipos: um de $3 \mathrm{~m}$ de lado e outro de $30 \mathrm{~m}$. Observe-se que, nessa mesma época, o físico norte-americano-escocês Ronald ("Ron”) William Prest Drever (1931-2017), na Escócia, também iniciou suas pesquisas com a proposta de Weber e usando também a IO.

Ainda na primeira metade da década de 1970, Weiss deu continuidade à sua pesquisa sobre a RCF e com, seu grupo de estudantes-pesquisadores, começou a medir a natureza térmica dessa radiação, em grandes altitudes e por intermédio de balões atmosféricos. Como a National Aeronautics and Space Administration (NASA) havia também iniciado seu interesse nessa radiação, no verão de 1975, Weiss foi até ao seu Comitê-Técnico para tentar um financiamento para suas pesquisas em cosmologia e gravitação, tendo a IO como peça principal. Como existia um grupo de pesquisadores no California Institute of Technology (CALTECH), que também trabalhava naquelas áreas de pesquisa, dentre eles o nobelista Thorne (que se bacharelou no CALTECH, em 1962, e se doutorou na PU, em 1965, com a Tese intitulada Geometrodynamics of Cylindrical Systems, sob a orientação de Wheeler), Weiss sugeriu ao

\footnotetext{
${ }^{57}$ Physical Review Letters, v. 22, p. 1320, 1669.
} 
comitê que o convidasse para opinar sobre sua proposta. Aceita sua indicação, Weiss foi recebê-lo em Washington e, então, por uma noite, Weiss teve a oportunidade de descrever seu IO para Thorne que, no entanto, ainda não havia pensado profundamente sobre essa possibilidade.

De volta ao CALTECH, Thorne sugeriu à sua administração que investisse na proposta de Weiss e, então, formou um grupo de pesquisa para desenvolver um projeto análogo. Como Barish (como vimos acima) havia tornado possível a construção e a operação do LIGO, assim formou-se o trio de nobelistas.

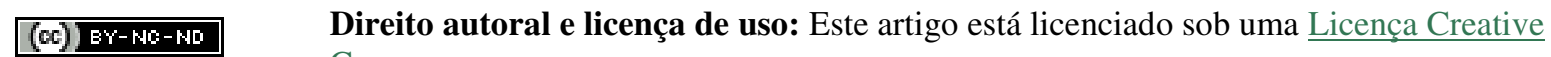
Commons. 